\title{
INFECÇÕES RELACIONADAS AO USO CATETER VENOSO CENTRAL: REVISÃO INTEGRATIVA
}

\section{CENTRAL VENOUS CATHETER-RELATED INFECTIONS: INTEGRATIVE REVIEW}

\author{
Kauana Aparecida Dionísio Freitas ${ }^{1}$; Emilli Karine Marcomini ${ }^{2}$; Nanci Verginia Kuster de \\ Paula ${ }^{3}$
}

Enfermeira graduada pela Universidade Paranaense. Pós-graduada em Unidade de Terapia Intensiva. Responsável Técnica na Universidade Paranaense ${ }^{1}$. Enfermeira graduada pela Universidade Paranaense. Mestranda em Biotecnologia pela Universidade Federal do Paraná. Bolsista CAPES ${ }^{2}$. Enfermeira graduada pela Pontifícia Universidade Católica do Paraná. Mestre em Engenharia de Produção pela Universidade Federal de Santa Catarina. Docente na Universidade Paranaense ${ }^{3}$.

\section{Abstract}

The aim of this study was to analyze the main causes of infections related to the central venous catheter in patients in the intensive care unit, to identify the conditions that alleviate or delay the evolution of infections and to evaluate nursing actions to prevent infections. It is an integrative literature review, carried out in the VHL and Google Scholar databases during the years 2014 to 2019. Of the eleven articles evaluated, three categories emerged to answer the proposed objectives. It was identified that the causes related to infections are associated with the introduction of the catheter, incorrect insertion and handling technique, absence of training, low adherence to hand hygiene, long stay and unnecessary dressing changes. The actions that reduce these causes are related to the use of barrier precautions, the use of 5\% chlorhexidine, hand hygiene and the use of bundles. In nursing care, it evidenced the practical adherence to the existing rules, daily assessment of the practice and application of aseptic assistance.

Keywords: Central Venous Catheterization; Nursing care; Catheter-related infections; Intensive Care Units.

\section{Resumo}

O objetivo deste estudo foi analisar as principais causas de infecções relacionadas ao cateter venoso central em pacientes da unidade de terapia intensiva, identificar as condições que amenizam ou retardam a evolução das infecções $e$ avaliar as ações de enfermagem para prevenção das infeç̧ões. Trata-se de uma revisão integrativa da literatura, realizada nas bases de dados BVS e Google Acadêmico durante os anos de 2014 a 2019. Dos onze artigos avaliados, emergiram três categorias para responder aos objetivos propostos. Identificou-se que as causas relacionadas as infecções se associam a introdução do cateter, técnica incorreta de inserção e manuseio, ausência de treinamentos, baixa adesão a higienização das mãos, longo tempo de permanência e troca de curativos desnecessário. As ações que reduzem essas causas se relacionam a utilização de precaução de barreira, uso da clorexidina 5\%, higienização das mãos e utilização de bundles. Nos cuidados de enfermagem, evidenciou a adesão prática das normas já existente, avaliação diária da prática e aplicação de assistência asséptica.

Palavras-chave: Cateterismo Venoso Central; Cuidados de Enfermagem; Infecções relacionadas a Cateter; Unidades de Terapia Intensiva. 


\section{Introdução}

O Cateter Venoso Central (CVC) consiste em um dispositivo invasivo para acesso ao sistema intravascular, bastante empregado na Unidade de Terapia Intensiva (UTI), com a finalidade de administração de medicamentos, nutrição parenteral e monitorização hemodinâmica ${ }^{1}$.

Mesmo diante dos benefícios ofertados pelo cateter, ressalta-se que há riscos relativos ao seu uso, especialmente pela possibilidade de desenvolvimento de infecção, elevando a morbimortalidade do paciente que muitas vezes já se encontra em um estado de vulnerabilidade ${ }^{1,2}$.

Pontua-se que a inadequação de cuidados assépticos com o dispositivo, a ausência de Equipamentos de Proteção Individual (EPI) e baixa prática de higienização das mãos, ocasiona danos ao paciente, permitindo a migração de microrganismos para dentro do sistema vascular, contribuindo para as Infecções da Corrente Sanguínea (ICS) associadas a $\mathrm{CVC}^{3}$.

Todos esses fatores anteriormente citados, quando aplicados, compõem ferramentas que elevam a segurança do paciente, a qualidade da assistência e reduzem os recursos financeiros ${ }^{3}$. Assim, cada instituição de saúde deve elaborar seu protocolo de cuidado para prevenção de ICS relacionada a cateteres e promover medidas aplicáveis direcionadas a todos os profissionais que lidam com o paciente em uso de $\mathrm{CVC}^{4}$.

Diante desse paradigma de infecção e sabendo da atuação do enfermeiro no ambiente intensivo, afirma-se que os cuidados de enfermagem são primordiais para redução de infecções, principalmente pela identificação de manifestações flogísticas e pelo manejo seguro de dispositivos intravasculares ${ }^{5}$.

Em razão dos enfermeiros estarem em maior contato com o paciente e de conhecer fatores que podem estar associados à infecção, eles compõem a peça chave para aplicar intervenções capazes de reduzir consideravelmente os riscos associados ao uso de $\mathrm{CVC}^{2,5}$.

Desta forma, o objetivo geral do presente estudo consiste em analisar quais as principais causas de infecções relacionadas ao uso de CVC em pacientes internados na Unidade de Terapia Intensiva (UTI), identificar as condições que amenizam ou retardam a evolução das infecções e avaliar as ações de enfermagem para prevenção das ICS.

\section{Metodologia}

Tipo de estudo

A metodologia empregada na então pesquisa foi a revisão integrativa da literatura, escolhida por permitir a reunião de informações, a avaliação crítica e a sintetize de estudos já existentes sobre o tema investigado, possibilitando uma conclusão a partir desses achados. A revisão integrativa proporciona a inclusão de estudos teóricos e de abordagens metodológicas distintas (qualitativas e quantitativas), empregando uma análise sistemática ${ }^{6}$.

\section{Critérios de seleção (inclusão e exclusão)}

A partir das seguintes questões: "quais as principais causas de infecção relacionada ao uso cateter venosa central?", "quais as condições que amenizam ou retardam as infecções?", "quais ações podem ser desempenhadas pela enfermagem na prevenção dessas infecções? foram identificadas palavras-chaves correspondentes às perguntas, por meio dos Descritores em Ciências da Saúde (DeCS).

Os critérios de inclusão utilizados foram: idioma na língua portuguesa, ano de publicação (2014-2019) e tipo de documento (artigo original e artigo de revisão). Foram excluídos artigos envolvendo estudos com animais, estudos teóricos/ reflexivos, dissertações, cartas ao editor e estudos que não estavam de acordo com os critérios de inclusão (que ainda apareceram após refinamento da busca).

Em seguida, após a leitura dos títulos e resumos dos artigos encontrados, foram descartados aqueles que estavam em duplicada e que não contemplavam o objetivo do estudo, a fim de, selecionar apenas os artigos que abordavam os temas de interesse da pesquisa. Por fim, os artigos foram novamente lidos na íntegra, onde se obteve a amostra final de artigos da pesquisa.

Coleta de dados - instrumentos e procedimentos de coleta

A busca ocorreu no mês de maio de 2019. Para a seleção dos artigos utilizou-se as bases de dados Literatura Latino-Americana e do Caribe em Ciências da Saúde (LILACS), Centro Latino-Americano e do Caribe de Informação em Ciências da Saúde (BIREME), Sistema Online de Busca e Análise de Literatura Médica (MEDLINE) e Base de Dados de Enfermagem (BDENF), 
acessadas por meio da Biblioteca Virtual em Saúde (BVS) e Google Acadêmico, aplicando quatro descritores (Cateterismo Venoso Central AND Cuidados de Enfermagem AND Infecções relacionadas a Cateter AND Unidades de Terapia Intensiva).

Procedimentos de análise e tratamento dos dados quanti e/ou qualitativos

Os artigos foram organizados em banco de dados de acordo com referência completa, país de origem dos autores e da revista, autores, objetivos, tipo do estudo, amostra e principais resultados encontrados.

A partir da análise dos conteúdos dos estudos, identificaram-se três categorias temáticas principais, sendo elas: Causas de infecções relacionadas ao uso cateter venoso central; Condições que amenizam ou retardam a evolução das infecções; Ações de enfermagem para a prevenção de infecções relacionadas ao uso de cateter venoso central.

\section{Aspectos éticos}

Por se tratar de uma pesquisa em que não envolve a manipulação de seres vivos de forma direta, não foi necessário o envio ao comitê de ética em pesquisas envolvendo seres humanos. No entanto, toda forma de cuidado quando ao referenciamento foi seguida para que não houvesse plágio, bem como para que as informações principais pudessem ser transcritas de forma adequada por meio das citações.

\section{Resultados}

As buscas nas bases de dados da Biblioteca Virtual em Saúde (BVS) e Google Acadêmico resultaram em onze publicações, sobre as quais procedeu-se a sistematização da revisão integrativa. A fim de facilitar 0 entendimento do processo de seleção dos artigos nas bases de dados elaborou-se o fluxograma 01 e para apresentar as informações dos estudos escolhidos elaborou-se a tabela 01, contendo as seguintes variáveis: título, autores, identificação da publicação e objetivo.

Fluxograma 1: Descrição detalhada da busca dos artigos

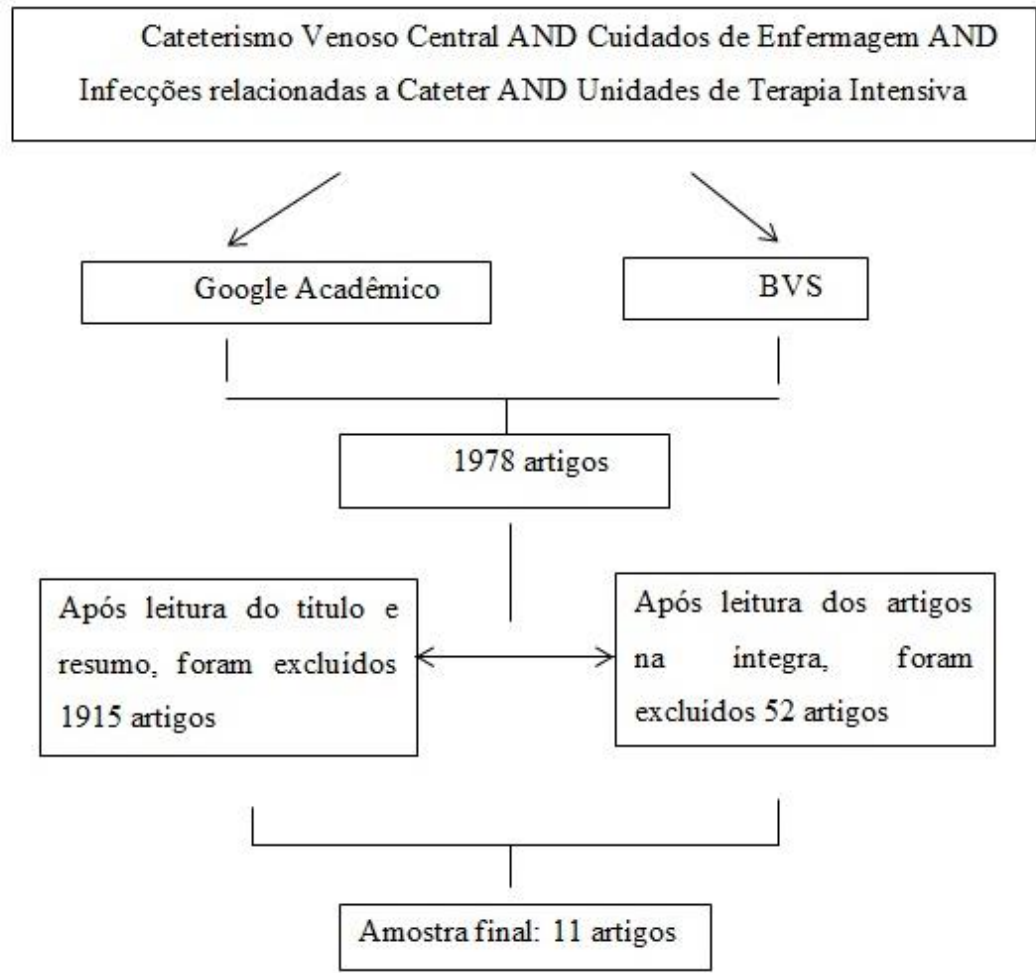


Tabela 1: Síntese dos estudos sobre infecções relacionadas ao uso cateter venoso central.

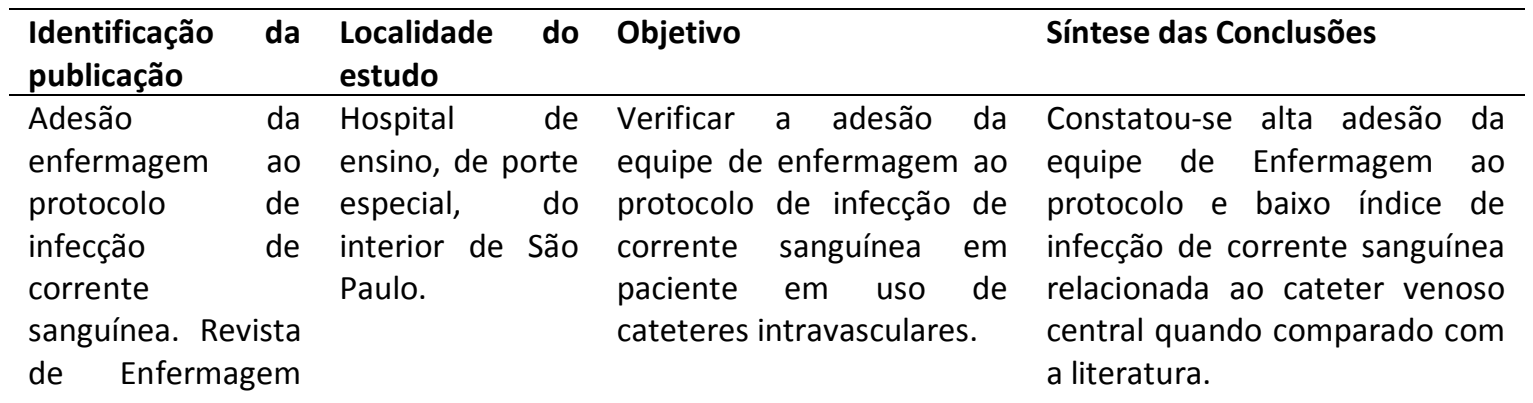

UFPE online. 2018; 12(09):2361-2367.

Adesão da equipe de enfermagem as medidas de prevenção de infecções de corrente sanguínea. Revista de Enfermagem UFPE online. 2017; 11(10):3698-3706.

Ações

enfermagem na

prevenção de

infecções

relacionadas ao

cateter venoso

central: uma

revisão integrativa.

Revista SOBECC.

2014; 19 (4):219-

225.

Fatores de risco para infecções da corrente sanguínea relacionadas a cateter em unidades de terapia intensiva pediátrica. Revista Brasileira Terapia Intensiva. 2018; 30(4): 436-442.

Tempo permanência do curativo gel de clorexidina no cateter venoso central em paciente crítico. Escola Anna Nery. 2017; 21(4).

Infecção na inserção do cateter venoso central. Rev. Pre. Infec e Saúde.

2015;
Hospital-Escola situado no município de Campina Grande (PB), Brasil.

Revisão integrativa na Biblioteca Cochrane e nas bases de dados Web of Science, CINAHL, MEDLINE, LILACS, BDENF, IBECS.

Hospitais da cidade de São Paulo.

UTI de um hospital de ensino de alta complexidade localizado no interior do estado de São Paulo.

Revisão integrativa nas bases MEDLINE, Web of Science, Elsevier.
Avaliar o conhecimento e adesão da equipe de enfermagem as medidas de prevenção de infecções de corrente sanguínea relacionadas ao cateter venoso central em unidade de terapia intensiva.

Identificar as ações de enfermagem para a prevenção de infecções primárias da corrente sanguínea.

Determinar os fatores de risco para contrair infecções da corrente sanguínea associadas a cateter de acesso central em unidades de terapia intensiva pediátrica e investigar a incidência e a etiologia dessas infecções.

Identificar o tempo de permanência do curativo gel de clorexidina aplicado no sitio de inserção do cateter venoso central, descrever motivos de troca e identificar a quantidade utilizada por pacientes internados UTI.

Reunir e sintetizar evidências disponíveis na literatura sobre a prevenção de infecções de corrente sanguínea
Conclui-se que a adesão às medidas de prevenção de ICSRCVC deve ser incentivada através de educação continuada.

As evidências sobre os cuidados de enfermagem para pacientes em uso de cateter venoso central servem de base para se realizar uma assistência efetiva, segura, de qualidade e com custos reduzidos.

Maior duração do uso de cateter venoso central e mais de um cateter venoso central de uma vez foram os principais fatores de risco para infecções da corrente sanguínea associadas a cateter em unidades de terapia intensiva pediátrica.

A permanência do curativo foi inferior a sete dias acarretando um número maior de curativo utilizado por paciente. 0 descolamento do curativo foi o motivo mais frequente de troca não programada.

Evidenciou que é de suma importância o cuidado na inserção de cateter venoso central para a prevenção de infecção de corrente sanguínea. 
1(3):46-54.

Implantação de

bundles em

unidade de terapia

intensiva: um

relato de

experiência. Rev

Enferm UFSM.

2014; 4(1):227-

236. Avaliação dos
cuidados de
enfermagem com
o cateter venoso
central em uma
unidade de terapia
intensiva adulto e pediátrica. Rev. Adm. Saúde. 2018; 18(70).

Impacto de dois bundles na infeção relacionada a cateter central em pacientes críticos. Revista LatinoAmericana de Enfermagem. 2017; 25 (1):1-7.

Adesão às medidas para prevenção da infeç̧ão da corrente sanguínea relacionada ao cateter venoso central. Enferm. Foco. 2017; 8 (2): 36-41.

relacionada à inserção de cateter venoso central.

Hospital público de parceria privada, localizado na cidade de São Paulo.

Hospital público de Minas Gerais.

Revisão integrativa de artigos publicados no Portal Capes, Biblioteca Virtual em Saúde, PubMed, Science Direct, Cochrane, CINAHL SCOPUS. Hospital público de Belo Horizonte, Minas Gerais.
Demonstrar o processo de implantação dos três bundles em unidade de terapia intensiva, relacionados à prevenção de pneumonia associada à ventilação mecânica, infecção de corrente sanguínea associada ao cateterismo venoso central e infecção do trato urinário associado à sondagem vesical de demora.

Avaliar os cuidados de enfermagem relacionados ao cateter venoso central (CVC) em uma Unidade de Terapia Intensiva adulto e pediátrica, comparar a conduta ao protocolo padronizado na instituição e analisar os resultados baseando-se no manual da Agência Nacional de Vigilância de 2017.

Avaliar o impacto da implementação de bundles de inserção e manutenção nas taxas de infecção da corrente sanguínea relacionada a cateter venoso central numa unidade de terapia intensiva.

Verificar a adesão da equipe multiprofissional para as medidas de prevenção da infecção da corrente sanguínea relacionada ao cateter venoso central.
Houve diminuição das infecções ao paciente após a implantação das medidas preventivas, com a otimização da qualidade e segurança. o apoio diretivo, disponibilização de recursos e engajamento dos enfermeiros gestores foram essenciais para o êxito na implantação dos bundles

A auditoria no cuidado de enfermagem na troca de curativo de CVC favoreceu um processo educativo entre os enfermeiros, contribuiu para identificar estratégias para melhorar a assistência e propôs uma atualização do protocolo da instituição.

Adoção de bundle evidenciou um impacto positivo na redução da infecção. Todavia, não se observou uma relação direta entre o número de medidas descritas nos estudos ou o maior tempo de implementação e taxas mais altas de redução da infecção.

Verificou-se uma baixa adesão global pelos enfermeiros e técnicos, especialmente à higiene das mãos $(22,7 \%)$ e desinfecção do hub $(10,4 \%)$ e uma adesão de $100 \%$ da equipe médica ao utilizar a barreira máxima de precaução. Conclusão: mesmo com um resultado satisfatório para a inserção dos cateteres nos períodos pré e pós-intervenção, essas medidas não representam completa adesão pela equipe de enfermagem.

Percepção dos Hospital Analisar a percepção dos Destaca-se

o




\begin{tabular}{lrcll}
\hline enfermeiros & Universitário & enfermeiros assistenciais & reconhecimento da Comissão \\
assistenciais sobre & no município de & $\begin{array}{l}\text { sobre a função da } \\
\text { como órgão preventivo, mas }\end{array}$ \\
a comissão de & Cajazeiras- & $\begin{array}{l}\text { Comissão de Controle de } \\
\text { muitas vezes atrelado somente }\end{array}$ \\
$\begin{array}{l}\text { controle } \\
\text { infecção }\end{array}$ & de & PB. & Infecção Hospitalar. & $\begin{array}{l}\text { à pós-exposição a acidentes de } \\
\text { trabalho, evidenciando a cultura }\end{array}$ \\
hospitalar. Enferm. & & & curativa no ambiente hospitalar.
\end{tabular}

Foco 2017; 8 (3):

56-60.

Considerando os periódicos de publicação científica dos artigos, observou-se que foram diversos, reforçando se tratar de uma temática de amplo interesse no meio científico. Em relação ao ano de publicação, os períodos de 2017 e 2018 obtiveram o maior índice de publicações com um percentual igualitário de $33,3 \%$. Nota-se ainda que o ano de 2016 , não houve publicações sobre a temática que se enquadrassem nos critérios de inclusão.

Todos os estudos selecionados são brasileiros, sendo que aproximadamente $40 \%$ foram realizados em hospitais do estado de São Paulo, seguido por estudos de revisão literária e pesquisas realizadas no estado de Pernambuco e Minas Gerais.

Após o preenchimento da Tabela 1, pode-se realizar uma leitura sistematizada das publicações e fazer uma análise crítica dos artigos, conseguindo-se dividir os resultados em três categorias temáticas, que serão discutidas abaixo.

\section{Discussão}

Causas de infecções relacionadas ao uso cateter venoso central

Os pacientes críticos que necessitam de internamento em uma unidade de alta complexidade devido à instabilidade orgânica são indispensáveis ao uso do cateter venoso central, em decorrência de sua importante utilização na assistência ${ }^{7}$. Em razão da inserção desse cateter ser em vasos de maior calibre, como é o caso das veias jugulares interna, subclávia e femoral ${ }^{2}$ e de sua ponta estar localizada próximo ou dentro do átrio direito do coração, eleva-se as possibilidade de desenvolvimento de uma infecção que comprometerá o quadro clínico do paciente ${ }^{7}$.

Assim, as causas das infecções relacionadas ao uso de cateter venoso central estão relacionadas com a não realização das técnicas corretas de instalação e manuseio do dispositivo, ocorrendo também em decorrência da ausência de treinamentos institucionais com a equipe multidisciplinar sobre os cuidados assépticos relativos ao cateter ${ }^{2}$.

Em um estudo realizado com profissionais de enfermagem que atuam em unidade de terapia intensiva, constatou que $90,9 \%$ dos entrevistados referiram que nunca receberam treinamentos sobre medidas de prevenção ICS, ressaltando ainda que o tema segurança do paciente tem baixa abordagem desde a formação ${ }^{8}$. Este fato interfere consequentemente na qualidade da assistência aplicada, pois a ausência de treinamentos pode levar a uma assistência precária que posteriormente levará ao aumento de infecções. Pode-se citar ainda que a problemática de infecção pode ser resolvida em $71 \%$, quando são seguidas rigorosamente as recomendações corretas de inserção e manutenção $\mathrm{CVC}^{9}$.

Outro fator de destaque para as causas de infecção, refere-se a baixa higienização das mãos seja para com o uso de álcool $70 \%$ ou água e sabão, onde verifica-se que os principais microrganismos causadores das infecções eram provenientes das mãos dos profissionais de saúde que manipularam este ${ }^{10}$.

Há ainda dois fatores de risco para infecção associada a CVC, sendo o longo tempo de permanência e a utilização de mais de um cateter. Tais fatores são bastante vivenciados no ambiente de terapia intensiva, principalmente em razão do longo período de internamento a qual o paciente percorre ${ }^{9}$.

Por fim, em se tratando dos curativos de CVC, uma pesquisa realizada em uma UTI do estado de São Paulo revelou que dos 64 cateteres existentes, foram realizados 159 curativos, das quais 83 trocas não foram programadas, ou seja, ocorreram antes de sete dias $^{7}$. Este alto índice de troca não programada pode estar relacionada a presença de umidade no local, sujidades visíveis e descolamento de fitas do curativo, elevando consequentemente a possibilidade de um processo infeccioso.

Outros estudos avaliaram técnicas de troca de curativo, identificando que em nenhuma das trocas realizadas pelo enfermeiro empregou- 
se técnica asséptica ao procedimento, o que implica em severos riscos ao paciente ${ }^{10}$.

\section{Condições que amenizam ou retardam a evolução das infecções}

A principal forma de reduzir as infecções relacionadas ao uso CVCs, se inicia desde a inserção do cateter, sendo necessária a utilização das precauções de barreiras, preparação da pele com clorexidina $0,5 \%$ e proteção estéril utilizando dos EPI, como gorro, máscara, avental, luvas e campos estéreis sobre o corpo paciente ${ }^{9}$.

Uma ação primordial na prevenção de infecções se refere a higienização das mãos, no qual consiste em uma atitude de forte impacto para a prevenção, principalmente quando realizada nos momentos principais da assistência ${ }^{8}$. O mesmo autor reforça ainda que a equipe de enfermagem apresenta vulnerabilidades na prevenção de ICS associadas a CVC, pois existe uma baixa quantidade de treinamentos e principalmente, seguimento das normas assépticas ${ }^{8}$.

Além disso, as ICS podem ser facilmente evitadas ou reduzidas com a utilização da esterilização, medidas de precaução padrão e técnicas assépticas eficazes ${ }^{11}$.

Em outro âmbito, a utilização de Bundles consiste em medidas efetivas para reduzir consideravelmente $o$ índice de infecções, elevando as chances de sobrevida do paciente, especialmente quando envolve os dois momentos cruciais da assistência, o de inserção e manutenção do cateter ${ }^{2,12,13}$.

Ações de enfermagem para a prevenção de infecções relacionadas ao uso do CVC

O enfermeiro é o profissional que está lado a lado do paciente durante todo o seu período de internamento, sendo, portanto, o que conhece as condições físicas e a necessidade de cuidados a serem prestados pela sua equipe. Por esta razão, ele necessita compreender os processos que originam uma ICS associada a CVC, desempenhando medidas para reduzir a ocorrência e aplicando uma assistência de mais qualidade e segurança $^{13}$

Durante a execução das atividades diárias, o enfermeiro tem um grande papel para minimizar as infecções, pois o mesmo é responsável pelos cuidados, manutenção e avaliação diária do paciente, sendo que a identificação de um sinal flogístico e a percepção de alterações sugestivas a quadro infeccioso, veem de sua competência ${ }^{2}$.
Neste âmbito, uma ação de enfermagem muito relevante na prevenção de infecções é a adesão aos protocolos de prevenção de infecção existentes, aos quais em sua maioria abordam a identificação de curativos e equipos, boas condições de higiene nos procedimentos e técnicas assépticas no cuidado ${ }^{14}$.

No entanto, um estudo avaliou que os profissionais de enfermagem não seguem as normatizações de higienização das mãos antes e após a realização dos procedimentos assistenciais, inserindo a necessidade de investimento na discussão e educação em saúde aos colaboradores, para prevenção da infecção da corrente sanguínea associada ao cateter ${ }^{15}$.

Outro ponto destaque refere-se ao nível de conhecimento dos enfermeiros sobre a atuação e função da comissão de controle de infeção, onde muitos não identifica a finalidade real deste grupo de controle, desta maneira, é fundamental que os profissionais cobrem e reconheçam a contribuição da comissão para a redução da infecção ${ }^{16}$.

Todavia, para que os profissionais de enfermagem consigam aplicar tais medidas de prevenção de infecções, há que se considerar também a necessidade de os serviços de gestão estarem interligados ao controle de infecção, promovendo medidas que elevem a segurança do paciente e a qualidade da assistência prestada ${ }^{8}$.

\section{Considerações finais}

A presente revisão integrativa permitiu identificar que existem poucas publicações atuais relacionadas a temática de CVC em UTI adulto, inserindo a necessidade de estudos mais amplos relacionados a temática.

Diante das causas das infecções relacionadas ao uso de cateter, destacou-se a não utilização de técnicas assépticas, baixa prática de higienização das mãos e uso de EPIs, longo tempo de permanência do dispositivo e poucos treinamentos e capacitações. Sendo assim, os serviços de saúde devem dispor de uma equipe que realize a educação continuada nas instituições hospitalares, apresentando os fatores que elevam a incidência de infecções bem como os que reduzem consideravelmente estas taxas. Além disso, deve haver profissionais que fiscalizem os serviços prestados pela equipe de saúde e cobrem o cumprimento dos protocolos de manejo ao cateter central.

O enfermeiro, como profissional atuante no ambiente intensivo deve propor medidas de identificação precoce dos fatores de risco de 
infecção, assim como, implementar intervenções capazes de garantir a segurança do paciente e a qualidade da assistência prestada.

Verifica-se que existem lacunas na prestação de assistência, aos quais podem levar ao desenvolvimento de infecções relacionadas ao cateterismo central, cabendo ao enfermeiro, primordialmente, instituir intervenções eficazes e educação continuada nos serviços intensivos.

\section{Referências}

1. Silva PR, Queiroz ALF, Buriti MEO, Ferreira MDT, Pinto ACMD. A importância do profissional da saúde na prevenção de infecção hospitalar causado por cateter venoso central. Mostra Interdisciplinar do Curso de Enfermagem. 2017;3(2).http://publicacoesacademicas.unicatoli caquixada.edu.br/index.php/mice/article/view/3 $\underline{287 / 2806}$

2. Santos SF, Viana RS, Alcoforado CLGC, Campos CC, Matos SSS, Ercole FF. Ações de enfermagem na prevenção de infecções relacionadas ao cateter venoso central: uma revisão integrativa. Rev. SOBECC. 2014; 19(4):219-225. https://doi.org/10.5327/Z14144425201400040008

3. Silva AG, Oliveira AC. Prevenção da infecção da corrente sanguínea relacionada ao cateter venoso central: Uma revisão integrativa. Vigil. sanit. Debate. 2016; 4 (2):117-125. https://doi.org/10.3395/2317-269x.00705

4. Perin DC. Evidências de cuidado para prevenção de infecção de corrente sanguínea relacionada a cateter venoso central: revisão sistemática sem metanálise. 125 p. (Dissertação). Universidade Federal de Santa Catarina. Florianópolis; 2015. http://dx.doi.org/10.1590/1518-8345.1233.2787 5. Dias EG. Perfil e atividades desempenhadas pelos profissionais de enfermagem na inserção e manutenção do cateter venoso central na unidade de terapia intensiva. Revista Saúde e Desenvolvimento. 2017;

11(7):2-

12.https://www.uninter.com/revistasaude/index. php/saudeDesenvolvimento/article/view/691/39 $\underline{9}$

6. Sousa LMM, Severino S, Vieira CM, Antunes V. Metodologia de Revisão Integrativa da Literatura em Enfermagem. Revista Investigação Enfermagem. 2017; 21:17-26. http://hdl.handle.net/20.500.12253/1311

7. Dolci ME, Margatho AS, Silveira RCCP. Tempo de permanência do curativo gel de clorexidina no cateter venoso central em paciente crítico. Esc Anna Nery. 2017; 21(4): e20170026. https://doi.org/10.1590/2177-9465ean-2017-0026

8. Dantas GD, Figueiredo DSTO, Nobre AMD, Pimentel ERS. Adesão da equipe de enfermagem às medidas de prevenção de infecções de corrente sanguínea. Rev enferm UFPE on line. 2017; 11(10):3698-706. https://doi.org/0.5205/reuol.12834-30982-1SM.1110201701

9. La Torre FP, Baldanzi G, Troster EJ. Fatores de risco para infecções da corrente sanguínea relacionadas a cateter em unidades de terapia intensiva pediátrica. Rev Bras Ter Intensiva. 2018; 30(4):436-442. https://doi.org/10.5935/0103-507x.20180066

10. Sousal FC, Pereira JC, Rezende DA, Chen L. Avaliação dos cuidados de enfermagem com o cateter venoso central em uma unidade de terapia intensiva adulto e pediátrica. Rev. Adm. Saúde.

2018;

18(70). http://dx.doi.org/10.23973/ras.70.92

11. Nascimento GC, Queiroz AAFLN, Sousa DM, Ibiapina ARS, Ferreira MCS, Moura MEB. Infecção na inserção do cateter venoso central. Rev. Pre. Infec e Saúde. 2015; 1(3):4654. https://doi.org/10.26694/repis.v1i3.4241

12. Shimahukuro PM, Paulon P, Feldmen LB. Implantação de bundles em unidade de terapia intensiva: um relato de experiência. Rev Enferm UFSM. 2014; 4(1):227-236. https://doi.org/10.5902/2179769211097

13. Fortunatti CFP. Impacto de dois bundles na infeção relacionada a cateter central em pacientes críticos. Revista Latino-Americana de Enfermagem. 2017; $25 \quad$ (e2951):1-7. https://doi.org/10.1590/1518-8345.2190.2951

14. Crivelaro N, Beccaria LM, Frutuoso IS, Silveira AM, Werneck AL. Adesão da enfermagem ao protocolo de infecção de corrente sanguínea. Rev. enferm. UFPE on line. 2018;

12(9):2361-

$2367 . \quad$ https://doi.org/10.5205/1981-8963v12i9a234886p2361-2367-2018

15. Silva $A G$, Oliveira $A C$. Adesão às medidas para prevenção da infecção da corrente sanguínea relacionada ao cateter venoso central. Enferm. Foco. 2017; 8 (2): 36-41. https://doi.org/10.21675/2357-

707X.2017.v8.n2.977

16. Félix TGS, Silva CRDV, Meira MLM, Negreiros RV, Mendes JMS, Véras GCB. Percepção dos enfermeiros assistenciais sobre a comissão de controle de infecção hospitalar. Enferm. Foco 2017; 8 (3): 56-60. https://doi.org/10.21675/2357707X.2017.v8.n3.1115 
Endereço para Correspondência

Emilli Karine Marcomini

Rua Maringá, 503, Francisco Alves.

Paraná - Brasil.

CEP: $87570-000$

E-mail: emillimarcomini@hotmail.com

Recebido em 06/09/2020

Aprovado em 19/03/2021

Publicado em 15/06/2021 